\title{
Dietary Obesity Linked to Genetic Loci on Chromosomes 9 and 15 in a Polygenic Mouse Model
}

\author{
David B. West, Jo Goudey-Lefevre, Barbara York, and Gary E. Truett \\ Obesity, Diabetes and Metabolism Section, Pennington Biomedical Research Center, Louisiana State University, \\ Baton Rouge, Louisiana 70808
}

\begin{abstract}
Loci linked to sensitivity to dietary obesity were identified by Quantitative Trait Locus (QTL) analysis of two mapping populations derived from a cross between $A K R / J$ and SWR/J mice. AKR/J mice are sensitive to dietary obesity when fed a high fat diet while SWR/J mice are resistant. Intercrosses between these strains segregate the phenotype of sensitivity to dietary obesity. Using an F2 mapping population of 931 male mice we found significant linkage with a QTL on chromosome 9 (Likelihood of the Odds [LOD] ratio of 4.85) and another QTL on chromosome 15 (LOD = 3.93). The presence of a QTL on chromosome 15 was confirmed in a separate mapping population of 375 male $F 1$ $\times S W R / J$ mice $(L O D=3.82)$. These two loci are designated dietary obese $2(\mathrm{Do} 2)$ and dietary obese $3(\mathrm{Do})$ for the chromosome 9 and 15 loci, respectively. Both of these chromosomal regions contain candidate genes which may contribute to variation in the phenotype. These loci also exert a significant control over individual adipose depot weights. (J. Clin. Invest. 1994. 94:1410-1416.) Key words: adipose • chromosome mapping • genetic models • linkage (genetics) - obese
\end{abstract}

\section{Introduction}

Obesity is an independent risk factor for a number of chronic diseases including noninsulin dependent diabetes mellitus, hypertension, and perhaps cardiovascular disease, in the human population (1). Because human obesity has a significant genetic component (2), identification of the genes involved in the expression of this disease would likely assist in the development of prevention and treatment strategies. However, the genetics of human obesity is complex. Multiple genes contribute to the trait $(3,4)$ and it is likely that there is significant genetic heterogeneity with environmental and lifestyle factors interacting with genetic predisposition to influence the amount of lipid stores. Of particular importance may be lifestyle factors such as the percentage of fat in the diet $(5,6)$ and the level of physical activity. This complex disease process will make identification of the genes contributing to obesity in human populations quite difficult.

Address correspondence to David B. West, Ph.D., Pennington Biomedical Research Center, 6400 Perkins Road, Baton Rouge, LA 70808.

Received for publication 8 March 1994 and in revised form 9 June 1994

J. Clin. Invest.

(C) The American Society for Clinical Investigation, Inc.

0021-9738/94/10/1410/07 \$2.00

Volume 94, October 1994, 1410-1416
An alternative approach to genetic studies in humans is to use an animal model with a similar phenotype to identify genetic mechanisms and then to use this information in directed studies of humans. Mice are ideal subjects for genetic studies due to the highly developed genetic map in this species (7) and their short generation time. Numerous rodent models of genetic obesity exist (8); however, the majority of these rodent obesities are caused by single gene mutations which result in massive obesity. Although it is likely that allelic variants at some of these single gene loci in rodents may contribute to polygenic obesity, it is also very likely that other genetic loci are involved. Therefore, studying only the single gene models of obesity in rodents may not give us a full picture of the potential genetic factors controlling body fat content especially those genes which modulate dietary obesity.

We have recently developed and characterized a polygenic model of differential susceptibility to dietary obesity in the mouse (9). The AKR/J strain of inbred mouse becomes significantly obese when it is fed a diet moderately high in fat calories while the SWR/J strain remains relatively lean. This increased sensitivity of $\mathrm{AKR} / \mathrm{J}$ mice to dietary obesity may not be dependent upon increased caloric intake; instead this strain difference may be due to differences in how dietary fat is metabolized. Segregation analysis of the progeny of intercrosses between these two strains show that this trait does segregate with a polygenic pattern of inheritance and with a minimum of 3 4 genetic loci controlling the trait (10).

Recently two new tools have been developed for mapping of the genes underlying complex phenotypes. The statistical approach known as quantitative trait locus (QTL) ${ }^{1}$ analysis has been developed to facilitate multipoint mapping of genetic loci that control a continuous quantitative phenotype in segregating populations $(11,12)$. This approach is significantly more powerful for detecting linkage than traditional single point analysis. The identification of highly polymorphic markers such as simple sequence length polymorphisms (SSLPs) which can be screened using polymerase chain reaction has also greatly accelerated the mapping of genes underlying complex genetic traits (13). The QTL mapping approach has been used successfully to map genetic loci controlling complex phenotypes in both plants (14) and animals $(15,16)$.

We have used this approach in a preliminary analysis of the segregation of the phenotype of sensitivity to dietary obesity in our mouse model. In an earlier report we have identified a locus on chromosome 4 linked to the phenotype of dietary obesity (10). We report here the identification of two additional genetic loci on chromosomes 9 and 15 which are linked to this trait.

1. Abbreviations used in this paper: LOD, likelihood of the odds; QTL quantitative trait locus; SSLP, simple sequence length polymorphism. 


\section{Methods}

Mapping populations. Inbred AKR/J and SWR/J mice (both mus musculus domesticus) were obtained from The Jackson Laboratory (Bar Harbor, ME) at $\sim 5$ wk of age. Genetic crosses were established between the parental strains using equal numbers of males and females from each strain. F1 generation mice were weaned at 28-35 d of age and were subsequently individually housed in plexiglass cages with ad libitum access to rodent chow (Purina Rodent Chow \#5001; Ralston Purina) and water. The F1 mice were either intercrossed or backcrossed onto SWR/J mice. For the backcross, equal numbers of male and female animals of both the parental strain (SWR/J) and the F1 population were used.

$\mathrm{F} 2$ and $\mathrm{F} 1 \times \mathrm{SWR} / \mathrm{J}$ litters containing four or more pups were used. If a litter contained more than 10 pups, the extra pups were randomly culled from the litter within 1 wk of birth. The sex of the pups culled was not determined. Litters containing fewer than four pups were discarded. Male pups ( $931 \mathrm{~F} 2$ and $375 \mathrm{~F} 1 \times \mathrm{SWR} / \mathrm{J}$ ) were weaned at 28 $32 \mathrm{~d}$ and placed into individual plexiglass cages and provided ad libitum access to rodent chow. Female pups were not utilized. The animals were kept in a room maintained at $22^{\circ} \mathrm{C}$ with a $12 \mathrm{~h}: 12 \mathrm{~h}$, light:dark cycle with lights on at $0700 \mathrm{~h}$.

Phenotyping. Between the ages of 35 and $42 \mathrm{~d}$, male mice were placed on a diet moderately high in fat content $(32.6 \mathrm{kcal} \%$ fat, 15.0 $\mathrm{kcal} \%$ protein, $52.4 \mathrm{kcal} \%$ carbohydrate; Diet \#C11024, Research Diets, Inc., New Brunswick, NJ) and maintained with ad libitum access to this diet for $12 \mathrm{wk}$. After cervical dislocation, the mice were dissected and the left and right inguinal, left and right epididymal, left and right retroperitoneal, and mesenteric adipose depots were removed and weighed. An index of adiposity was calculated by dividing the summed weight of the seven excised adipose depots by the eviscerated carcass weight (not including the dissected depots). We have previously shown that this adiposity index is highly linearly correlated with carcass lipid content with a correlation coefficient of 0.97 (16a). An adiposity index of 0.15 corresponds to a percent carcass lipid content of $\sim 30 \%$. The phenotypes which we used for the analysis reported in this paper include body weight, adiposity index, average weight of bilateral adipose depots (inguinal, epididymal, and retroperitoneal) and the mesenteric depot weight.

Genotyping. Genomic DNA was isolated from spleen using phenolchloroform extraction. Samples were homogenized in $10 \mathrm{mM}$ Tris/10 mM EDTA and cell membranes lysed by addition of Triton X-100 to $2 \%$. The nuclear pellet was resuspended in $10 \mathrm{mM}$ Tris/10 mM EDTA, lysed by addition of sodium dodecyl sulfate to $2 \%$ and digested overnight with proteinase $\mathrm{K}(100-500 \mathrm{microgram} / \mathrm{ml})$ at $65^{\circ} \mathrm{C}$. DNA was subsequently extracted stepwise with 1 volume of phenol, $\mathrm{pH} 8.0,1$ vol of phenol:chloroform (1:1) and 1 vol of chloroform. DNA was precipitated by addition of ammonium acetate to $2 \mathrm{M}$ and $1 \mathrm{vol}$ of isopropanol. The DNA strands were drained, redissolved in $10 \mathrm{mM}$ Tris $/ 1 \mathrm{mM}$ EDTA and quantified either by absorbance at $260 \mathrm{~nm}$ or fluorometrically using the dye $\mathrm{H} 33258$ (17).

Genomic DNA was used for genotyping at simple sequence length polymorphisms (SSLP) as described by Dietrich et al. (13). We used primers available through Research Genetics (Huntsville, AL) which have previously been mapped in the mouse. Since product size for SSLPs have not been described for the SWR/J strain, we screened a selection of those available in order to identify SSLPs polymorphic between the two parental strains. The SSLP products were amplified by using Taq DNA polymerase and 18-22 oligonucleotide primers flanking the SSLP in a total reaction volume of $20 \mu$ l. Each reaction contained $2.5 \mathrm{mM} \mathrm{MgCl} 2,100 \mu \mathrm{M}$ dNTPs, $130 \mathrm{nM}$ SSLP primers (Research Genetics, Huntsville, AL), $0.35 \mathrm{U}$ Taq polymerase (Promega, Madison, WI) and $50 \mathrm{ng}$ genomic DNA. After a $3-\mathrm{min}$ incubation at $94^{\circ} \mathrm{C}$, the samples were run through 30 cycles of $30 \mathrm{~s}$ at $94^{\circ} \mathrm{C}, 120 \mathrm{~s}$ at $55^{\circ} \mathrm{C}$, and $120 \mathrm{~s}$ at $72^{\circ} \mathrm{C}$. The last cycle was followed by a $7-\min$ period at $72^{\circ} \mathrm{C}$ and the samples were then cooled to $4^{\circ} \mathrm{C}$ until frozen. Samples were cycled in a 96-well microtiter plate using an MJ Research thermal cycler (Model PTC-100; MJ Research, Watertown, MA).
Sizing of PCR products was generally performed by loading 15 $\mu \mathrm{l}$ of product on $5-6 \%$ agarose (NuSieve GTG; FMC BioProducts, Rockland, ME) mini slab gels and running at $4-6 \mathrm{~V} / \mathrm{cm}$ for $2-4 \mathrm{~h}$. Products were visualized by ethidium bromide staining. Some products were sized using $5 \%$ nondenaturing polyacrylamide sequencing gels with silver staining (Silver Sequence; Promega) to visualize products.

A total of 63 markers have been used in our scan of the mouse genome. The markers comprise 14 linkage groups on 12 chromosomes. This marker set does not include markers on either the $\mathrm{X}$ or $\mathrm{Y}$ chromosomes. However, through analysis of the breeding records for the F2 population we were able to identify the donor strain for the Y chromosome in each individual in the F2 population. Similarly, examining the breeding records in the $\mathrm{F} 1 \times \mathrm{SWR} / \mathrm{J}$ mapping population we were able to identify the strain source of the $\mathrm{X}$ chromosome in each individual. According to this analysis, neither the $\mathrm{X}$ nor the $\mathrm{Y}$ chromosome contain loci affecting the adiposity trait in these two populations.

Statistical analysis. The general statistical approach used for analyzing the linkage data was interval mapping, also known as mapping of quantitative trait loci $(11,12,18)$. For the 931 animals in the $F 2$ populations, only those in the $10 \%$ tails of the adiposity index phenotype distribution were genotyped $(n=189)$. Genotyping of only the extremes of the distribution has been described by Lander and Botstein (18) for mapping of QTLs. Compared with genotyping a relatively small mapping population across the entire phenotype distribution, this approach significantly improves the statistical power and the likelihood of finding linkage. Furthermore, it reduces the number of animals which must be genotyped if a large mapping population is available.

To confirm the presence of a QTL at a particular location, the $5 \%$ tails of the phenotype distribution were genotyped in the 375 F1 $\times$ SWR/J population. The F1 $\times$ SWR/J cross was used only to confirm the QTLs first identified in the F2 mapping population. We used F1s backcrossed on the lean strain (SWR/J) because previous work had suggested that there was a partial dominance for controlling body fat content of the AKR/J genotype (10). Therefore, using this backcross it was more likely that we would detect QTLs than if we used the backcross on the dominant or obese strain $(\mathrm{AKR} / \mathrm{J})$.

The statistical program Mapmaker/QTL (19) (generously provided by S. Lincoln and E. Lander of the Whitehead Laboratories at the Massachusetts Institute of Technology) was used to analyze the F2 and backcross data. This program performs a multipoint interval analysis across linkage groups and calculates the likelihood of the odds (LOD) score at specified intervals. This LOD score gives the $\log _{10}$ of the likelihood that the data reflect the presence of a locus versus the absence of a locus linked to the phenotype. A LOD score of greater than 3.0 is generally accepted as significant evidence of a QTL. However, it should be noted that this program does not correct for multiple tests for linkage. Although only the 10 or $5 \%$ extremes of the mapping populations were genotyped, values for the entire adiposity phenotype distribution were used in the statistical analysis. For comparison of phenotypes after grouping by genotype we used a nonparametric statistic, the Kruskal Wallis ANOVA, with the $P$ value set at .05 for statistical significance.

\section{Results}

Adiposity index in the F2 mapping population. Fig. 1 depicts the frequency distribution of the adiposity index phenotype in the 931 F2 animals used in this study along with the phenotype range for the two parental strains. The level of adiposity clearly segregated in this population with the leanest $\mathrm{F} 2$ reaching the level of the leanest SWR/J mice and the fattest F2 exceeding the levels of the most obese $\mathrm{AKR} / \mathrm{J}$ mice. The distribution is approximately normal with a mean value of 0.12 adiposity units, skewness of -0.29 and kurtosis of -0.26 . The adiposity phenotype used in the QTL analysis was unadjusted raw data.

Loci for total adiposity located on chromosomes 9 and 15. Using the F2 mapping population, two QTLs were identified 


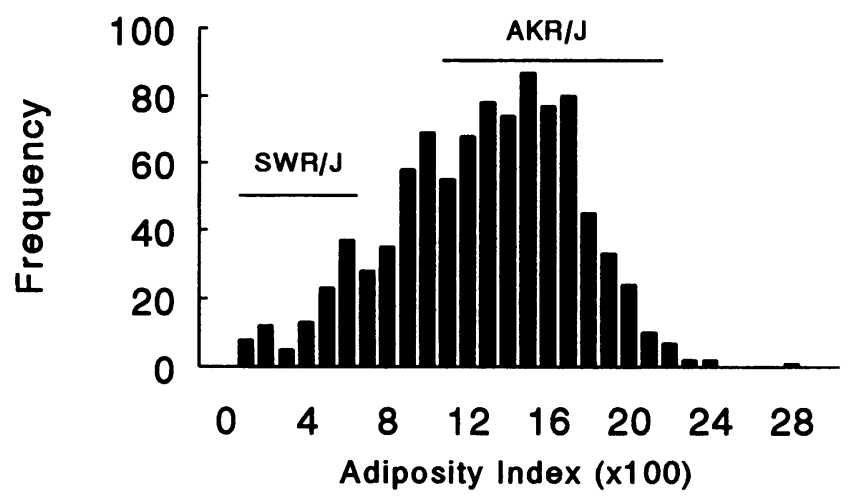

Figure 1. Frequency distribution of the adiposity index phenotype in 931 male $\mathrm{F} 2$ animals derived from an AKR/J $\times \mathrm{SWR} / \mathrm{J}$ cross and used for mapping of QTLs in this report. For reference, the ranges of the phenotype in the AKR/J and SWR/J parental strains are shown by the labelled horizontal bars.

with significant LOD scores for total adiposity. On chromosome 9 we identified a QTL with a peak LOD score of 4.85 (unconstrained genetic model) located between markers D9Mit18 and D9Mit11. For the chromosome 9 locus, the LOD distribution for linkage within this QTL is depicted in Fig. 2. A model with the AKR/J allele dominant for the chromosome 9 QTL can be rejected since the probability for this dominant model is more than a log unit lower than both an additive model and a recessive

\section{Chromosome 9}

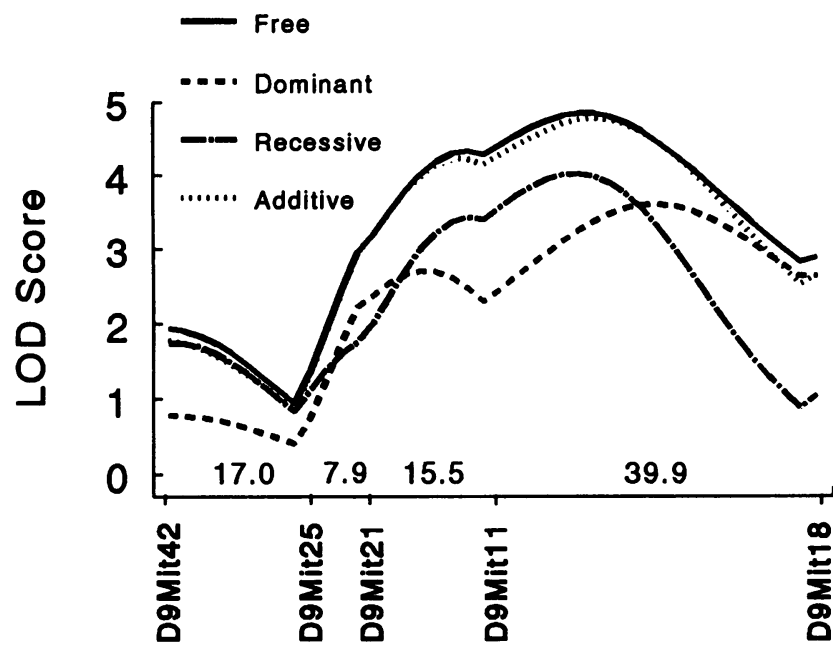

Figure 2. LOD ratio on chromosome 9 for total body adiposity in F2 progeny of an intercross between $S W R / J$ and $A K R / J$ mice. The abscissa labels indicate the SSLP markers used for the linkage analysis and the numbers directly above the abscissa indicate the distance between adjacent markers in centimorgans based upon recombination fractions. The LOD score was calculated by the MAPMAKER/QTL program at 2 centimorgan intervals spanning the distance between each pair of polymorphic markers. Four genetic models were used: free or unconstrained, AKR/J allele dominant, AKR/J allele recessive, and additive. A LOD score of $>3.0$ is generally accepted as significant. Since the maximum LOD score of the AKR/J dominant model is more than one $\log _{10}$ unit below that of the unconstrained model, the dominant model of inheritance can be rejected. This QTL on chromosome 9 linked to sensitivity to dietary obesity has been termed dietary obese 2 ( Do2 $)$.

\section{Chromosome 15}

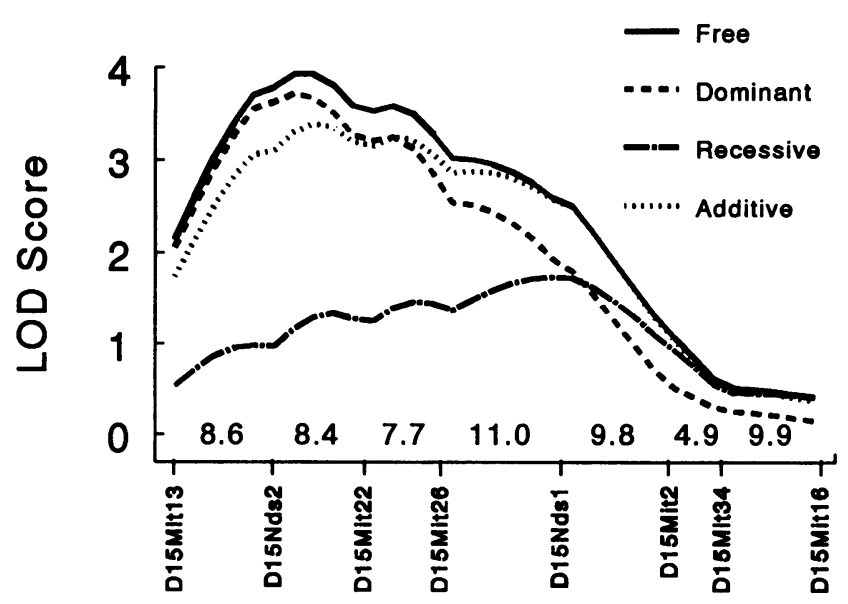

Figure 3. LOD ratio on chromosome 15 for total body adiposity in $\mathrm{F} 2$ progeny of an intercross between $S W R / J$ and AKR/J mice. Labelling of abscissa and different genetic models as in Fig. 2. The AKR/J recessive model can be rejected. This QTL on chromosome 15 linked to dietary obesity has been termed dietary obese $3(D o 3)$.

model. On chromosome 15 we identified another significant QTL with a peak LOD score of 3.93 (unconstrained genetic model) located between markers D15Nds2 and D15Mit22. A model with $A K R / J$ alleles recessive can be rejected for the inheritance of the QTL on chromosome 15 since both a dominant model and an additive model have a probability of more than one log unit greater than a recessive model. This QTL on chromosome 15 is depicted in Fig. 3. Since we had previously identified a locus on chromosome 4 linked to dietary obesity which we have named Dol (10), we have named these two newly identified loci linked with dietary obesity as dietary obese $2(D o 2)$ and dietary obese $3(D o 3)$ for the chromosome 9 and 15 loci, respectively.

Checking the $\mathrm{F} 1 \times \mathrm{SWR} / \mathrm{J}$ mapping population for linkage with the adiposity index at these same chromosomal locations showed that there was no significant linkage of adiposity with markers on chromosome 9. However, on chromosome 15 in the $\mathrm{F} 1 \times \mathrm{SWR} / \mathrm{J}$ population we found significant linkage at the same location as found in the F2 mapping population with a peak LOD score of 3.82 (data not shown).

Loci for individual adipose depots on chromosomes 9 and 15. In general, all of the individual adipose depots had significant LOD scores (LODs > 3.0) at the QTLs for total adiposity on chromosomes 9 and 15 (Table I) and the peak LOD scores for individual depots were in approximately the same position as the peak LOD scores for total adiposity (data not shown). In addition, total body weight had a marginally significant LOD score for both the chromosome 9 and 15 loci linked to total adiposity and individual adipose depot weight (see Table I). The locus on chromosome 9 had a highly significant LOD value for mesenteric adipose depot weight with the peak LOD score located between D9Mit 18 and D9Mit11.

Effect of chromosome 9 and 15 genotype on total adiposity. The effect of genotype on adiposity in the F2 mapping population at the D9Mit18 SSLP on chromosome 9 and the D15Mit22 SSLP on chromosome 15 is shown in Fig. 4. The chromosome 
Table I. Lod* Scores for Body Weight, Adiposity Index, and Adipose Depot Weight

\begin{tabular}{|c|c|c|}
\hline & \multicolumn{2}{|c|}{ Maximum LOD } \\
\hline & $\begin{array}{c}\text { Chromosome } 9 \\
(D o 2)^{\ddagger}\end{array}$ & $\begin{array}{c}\text { Chromosome } 15 \\
(D o 3)^{\ddagger}\end{array}$ \\
\hline Body weight (g) & 2.75 & 3.12 \\
\hline Adiposity index ${ }^{8}$ & 4.85 & 3.93 \\
\hline Inguinal depot (g) $)^{\|}$ & 3.39 & 3.83 \\
\hline Epididymal depot (g)" & 5.39 & 3.40 \\
\hline Retroperitoneal depot (g)" & 4.36 & 4.29 \\
\hline Mesenteric depot (g) & 14.73 & 4.11 \\
\hline
\end{tabular}

* The LOD score is the $\log _{10}$ of the likelihood of the odds ratio that the data represent the presence of a linked locus versus the absence of a linked locus at that location. ${ }^{\ddagger} D o 2$ and $D o 3$ refer to the loci on chromosomes 9 and 15, respectively, which are linked to the adiposity index. 'The adiposity index is calculated by dividing the summed weight of seven adipose depots by the eviscerated carcass weight. "Average of left and right depots.

9 allele from the SWR/J mice was clearly associated with a greater adiposity in the F2 mice. Animals with both alleles from the SWR/J parental strain at the chromosome 9 SSLP were clearly fatter than those with one SWR/J allele or two AKR/J alleles. As shown in Fig. 2, this is consistent with either an additive or an $\mathrm{AKR} / \mathrm{J}$ recessive model of inheritance contributing to the adiposity phenotype in the $\mathrm{F} 2$ population at the $\mathrm{Do} 2$ locus. Mice with either one or two AKR/J strain alleles at the chromosome 15 locus had higher adiposity indices than those which were homozygous $\mathrm{SWR} / \mathrm{J}$ at this locus. This is consistent with an analysis of the QTL showing that the Do3 data at the chromosome 15 locus fit an additive or $\mathrm{AKR} / \mathrm{J}$ allele dominant model of inheritance.

D9Mit18
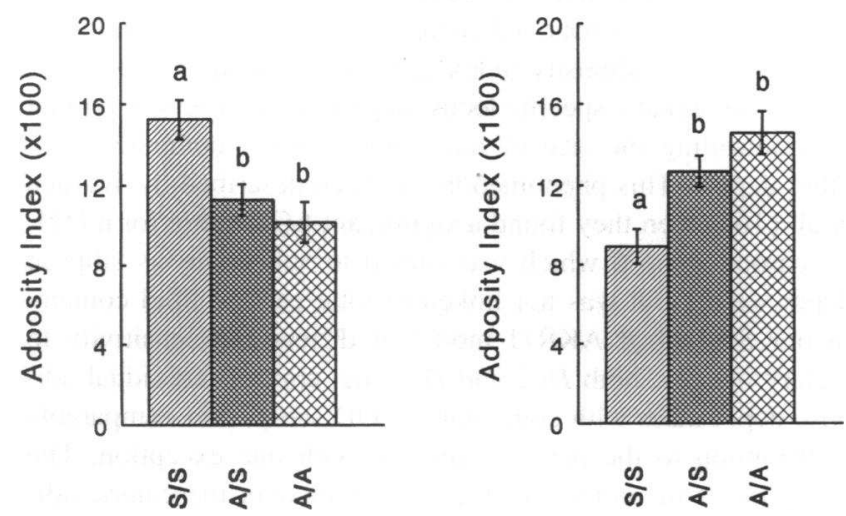

Figure 4. Effect of genotype at the D9Mit18 (chromosome 9) SSLP and the D15Mit22 (chromosome 15) SSLP on adiposity index in the F2 population. The data are from animals selected from the $10 \%$ extremes of the adiposity index distribution. In the figures, data are presented as means \pm standard errors. Bars labeled with different letters are significantly different from one another $(P<.05$; Kruskal Wallis ANOVA).

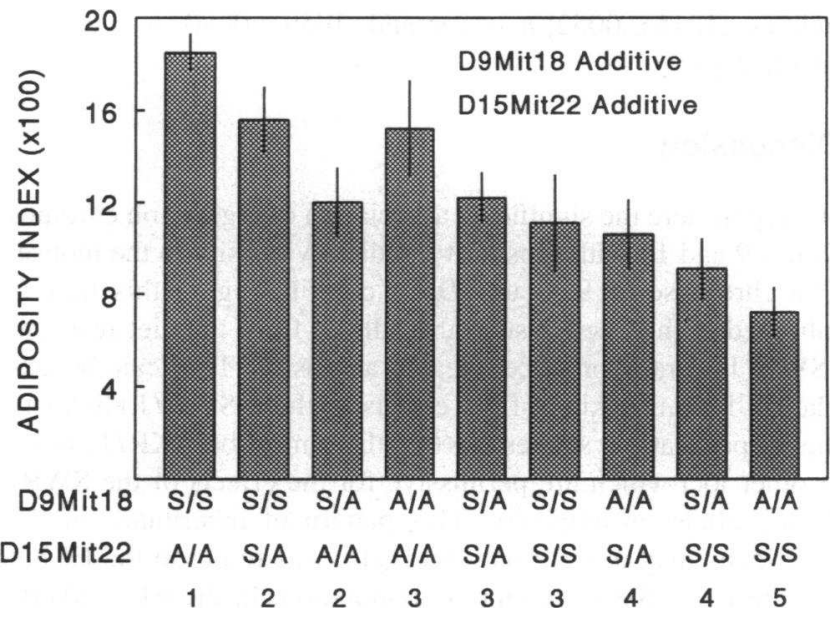

Figure 5. Effect of the nine different possible combinations of genotypes at the D9Mit18 (chromosome 9) and D15Mit22 (chromosome 15) loci on adiposity index in animals selected from the $10 \%$ extremes of the adiposity index distribution. Labels below the abscissa indicate the genotype at these two SSLP markers. S represents the SWR/J allele and A represents the AKR/J allele. Animals which are homozygous for the SWR/J allele at the chromosome 9 locus and homozygous for the AKR/ $\mathrm{J}$ allele at the chromosome 15 locus have nearly twice the adiposity of those animals which are homozygous AKR/J at the chromosome 9 locus and homozygous SWR/J at the chromosome 15 locus. The numerical values below the abscissa indicate the expected rank order of adiposity from highest to lowest assuming additive genetics for the two loci with the SWR/J allele increasing adiposity at the chromosome 9 locus and the AKR/J allele increasing adiposity at the chromosome 15 locus. This model further assumes that the effect of each locus on the phenotype is approximately the same magnitude.

Fig. 5 depicts the effect of combining the genotypes at these two loci on total adiposity. This model assumes the allele for D9Mit18 from the SWR/J parental strain is associated with greater levels of adiposity with additive genetics in the F2 population; and the allele from the AKR/J strain at the D15Mit22 locus also is associated with greater adiposity in the F2 population with additive genetics. It further assumes that the quantitative effect of the chromosome 9 and 15 loci on the adiposity phenotype is approximately the same. Mice which were homozygous SWR/J at the D9Mit18 locus, and homozygous AKR/ $\mathrm{J}$ at the D15Mit22 locus had an adiposity index approximately twice that of the mice which were homozygous AKR/J at the D9Mit 18 locus and homozygous SWR/J at the D15Mit22 locus.

To further evaluate the effect of the chromosome 9 and 15 loci on the adiposity index, we also genotyped, at the D9Mit18 and D15Mit22 markers, 146 animals randomly selected from the F2 population. At the D9Mit18 locus there was a slight but not significant effect of genotype on adiposity. Animals which were homozygous SWR/J genotype had an adiposity index of $.1264 \pm .0096(n=33)$; while heterozygous and homozygous $\mathrm{AKR} / \mathrm{J}$ mice had adiposity indices of $.1239 \pm .0052(n=73)$ and $.1186 \pm .0060(n=37)$ respectively. In contrast, at the D15Mit22 locus, there was a very large and significant effect of genotype on the adiposity index in this analysis of the crosssection of F2 animals $(P<.01 ;$ ANOVA $)$. Animals which had two $\mathrm{AKR} / \mathrm{J}$ alleles at the D15Mit22 locus were significantly fatter (adiposity index $=.1414 \pm .0075 ; n=34$ ) than animals having only one AKR/J allele or no AKR/J alleles (adiposity 
indices: $.1215 \pm .0052, n=78$; and $.1059 \pm .0080, n=31$, respectively).

\section{Discussion}

We report here the significant association of regions on chromosomes 9 and 15 with sensitivity to dietary obesity in the mouse. The chromosome 9 locus (Do2) contributing to this trait is inherited with genetics such that alleles from the diet resistant $(\mathrm{SWR} / \mathrm{J})$ strain produce greater adiposity. This can be explained by unmasking of the effects of these SWR/J alleles in the $F 2$ population, suggesting that there must be $A K R / J$ alleles at other loci which are permissive for the effects of the SWR/ $\mathrm{J}$ Do2 allele on adiposity. This pattern of inheritance of the Do2 locus may explain why this QTL was found in the F2 but not the F1 $\times \mathrm{SWR} / \mathrm{J}$ mapping population. In the F1 $\times \mathrm{SWR} /$ $\mathrm{J}$ population, $75 \%$ of all alleles are derived from the SWR/J parental line. Therefore, the appropriate genotypes at other loci necessary to unmask the phenotypic effects of the SWR/J allele at the Do2 chromosome 9 locus may not have occurred at a high enough frequency in the $F 1 \times S W R / J$ population.

The unmasking of the phenotypic effect of the SWR/J allele at the Do2 locus in the segregating F2 population is consistent with the genetic control of quantitative traits in plant populations. It has been reported in one experiment mapping the QTLs controlling 11 quantitative traits in the tomato that the parental phenotype did not predict the allelic effects in approximately $36 \%$ of the QTLs (20). In other words, the parental alleles had the opposite effect of that predicted (based upon the parental phenotype) in the segregating population. It is likely that the genetic control of polygenic traits in both animals and plants is quite complex with epistasis, modifier genes, major genes, and genes with recessive, dominant or additive genetics contributing to control of the phenotype. In addition, environment will certainly influence the effect that these genes have on the phenotype. The identification of additional QTLs controlling body fat content under varying dietary conditions will certainly be important in gaining a full understanding of the genetic factors controlling this phenotype in mice and perhaps in humans.

The AKR/J alleles at the chromosome 15 locus (Do3) contribute to increased adiposity with either a dominant or additive pattern of inheritance. The Do3 locus was identified in both the $\mathrm{F} 2$ and the $\mathrm{F} 1 \times \mathrm{SWR} / \mathrm{J}$ mapping populations at approximately the same position and with approximately equivalent LOD scores. The identification of the same locus in two separate mapping populations provides strong support for a locus at this location on chromosome 15 in the mouse controlling the response of body fat to manipulations of dietary fat.

The MAPMAKER/QTL program can be used to estimate the percent of the variance in a quantitative trait controlled by a particular locus or combination of loci. For the adiposity index in the F2 mapping population, the Do2 locus controls $6.9 \%$ of the variance while the Do3 locus controls $4.0 \%$ of the variance. Fixing the location of both of these loci gives a LOD score of 8.32 and explains $9.8 \%$ of the phenotype variance. However, since we genotyped only the $10 \%$ tails of the phenotype distribution this estimate of the percent of the variance controlled by these loci may not be accurate. Animals in the upper end of the phenotype distribution will be relatively enriched for alleles at multiple loci which promote increased body fat content. Similarly, animals at the lower extreme of the phenotype distribution will be enriched for alleles which are associated with low body fat content. Therefore, estimates of the variance controlled by a specific locus may not be precise since it is not likely that all combinations of genotypes affecting the trait are represented in the extremes of the phenotype distribution. A more precise determination of the percent of the phenotype variation controlled by the Do2 and Do3 loci must await either the genotyping of the entire phenotype distribution or identification of the other loci affecting the trait and controlling for their effects in the statistical model.

The effect of genotype at two SSLPs on chromosome 9 and 15 on the adiposity phenotype is profound (see Figs. 4 and 5). It is clear that the genotypes of individuals at these loci has significant value in predicting the degree of body fat content. The data for Fig. 4 were generated by genotyping only animals selected from the $10 \%$ tails of the phenotype distribution. As discussed above, the statistical approach of using the extremes of the distribution may exaggerate the effect of individual loci on the phenotype. To preliminarily address this we also genotyped individuals randomly selected from the F2 population at these same two SSLPs on chromosome 9 and 15. For the D15Mit22 marker, The AKR/J allele still had a very significant effect on the adiposity index in the cross-section of the F2 population. Animals homozygous for the AKR/J allele at the D15Mit22 marker were $33 \%$ fatter than animals homozygous for the SWR/J allele at this marker. This supports a significant role for the Do3 locus on chromosome 15 in controlling body fat content in this cross and further suggests that the percent of the variance controlled by this locus may be underestimated by using only the tails of the phenotype distribution in the analysis. In contrast, when the effect of genotype at the D9Mit 18 marker was assessed in this cross-section of the $F 2$ population, although the effect of genotype at this marker was in the same direction as that observed in animals from the tails of the distribution, the effect was smaller and not statistically significant. This further supports our conclusion that epistasis between alleles from the $\mathrm{SWR} / \mathrm{J}$ strain at this Do2 locus and AKR/J alleles at another locus or multiple loci are necessary for expression of the effects of the Do2 locus on body fat content.

Both the Do2 and Do3 loci are linked with individual depot weights as well as with total adiposity. This would be expected since the adiposity index is calculated from the summed weight of the dissected depots and therefore the weight of individual depots and the adiposity index are not independent. However, it is possible that a specific locus may be more or less important for controlling the size of one adipose depot compared with other depots. This phenomenon has been described by Warden et al. (16) when they found a significant LOD score for a QTL on chromosome 6 which was linked to subcutaneous adipose depot weight but was not linked to total carcass lipid content. In our $S W R / J \times A K R / J$ model of differential sensitivity to dietary obesity, both $\mathrm{Do} 2$ and $\mathrm{Do} 3$ loci control individual adipose depot mass with comparable LOD scores and comparable contribution to the percent variance with one exception. The exception is the control of the Do2 locus over mesenteric adipose depot weight. This locus controls $47 \%$ of the mesenteric depot weight variance and has a LOD score of 14.7. This suggests that the Do2 locus may be of greater relative importance for determining mesenteric depot weight than other depot weights. This information has significance since an understanding of the genetic factors which control intraabdominal fat mass may provide some insights into the mechanisms by which cen- 
tral fat distribution is associated with a significantly greater risk for the development of obesity-related diseases (21).

The polygenic model described in this report is different in a number of aspects from another model of polygenic obesity which has recently been described, the BSB mouse (22). The BSB mouse model is derived from an intercross of C57BL/6J mice crossed with Mus spretus and the F1 mice subsequently backcrossed onto the $\mathrm{C} 57 \mathrm{BL} / 6 \mathrm{~J}$ strain. A significant percentage of the mice in the backcross generation become obese spontaneously on a low-fat diet while neither of the parental strains demonstrate spontaneous obesity. In contrast, in the SWR/J $\times A K R / J$ cross neither of the parental strains or the $F 2$ animals become obese in the absence of a high fat diet. A QTL on chromosome 7 has been found to be linked to carcass lipid content in this BSB model (16). We have examined this same area of chromosome 7 in the AKR/J $\times S W R / J$ mouse model and have not found a QTL linked with dietary obesity. The transgression of the phenotype in the backcross progeny in the BSB model suggest some complex gene interactions producing obesity, analogous to the unmasking of the phenotype effects of the Do2 locus on chromosome 9 in the present report describing QTLs in the $A K R / J \times S W R / J$ cross. Given the limited number of genes which likely control body fat content in the mouse, some of the genes operating to control this phenotype are probably the same in these two models while others are likely to be different.

A number of candidate genes have been mapped to the regions of the Do2 and Do3 QTLs. On chromosome 9, Gnai2 , one of the alpha inhibitory subunits of $G$ protein, has been mapped to within 10 centimorgans of our peak LOD for adiposity between the D9Mit11 and D9Mit18 markers (23). The G protein is of particular interest since it modulates second messenger formation in the majority of hormonal and neurotransmitter systems (24) and therefore a defect in G protein function could be involved in many different phenotypes. Support for a role of altered $\mathrm{G}$ protein function in obesity comes from studies of pseudohypoparathyroidism in humans. This disease is due to a mutation in the $\mathrm{G}_{\text {s-alpha }}$ structural gene (25) and the complex phenotype of this disease includes early onset obesity. The alpha inhibitory subunit which maps to the Do2 QTL is expressed in mouse adipocytes (26) and is important in modulating alpha-2 adrenergic inhibition of lipolysis in adipocytes. The action of alpha-2 agonists on adipocytes can be blocked by pertussis toxin which ADP-ribosylates the alpha inhibitory subunit and uncouples the $G$ protein from the receptor (27). Therefore, modulation of the activity/function of this subunit may also have an impact upon adipocyte metabolism and adiposity.

The growth hormone receptor ( $\mathrm{Ghr}$ ) has been mapped to the proximal end of chromosome 15, at the location of Do3 $(28,29)$. There is a significant literature showing that manipulations of circulating growth hormone levels can have significant effects upon body composition in humans (30) and in animals $(31,32)$. Furthermore, defects in growth hormone receptor function have been shown to be related to obesity in human Laron dwarfism (33). Since the Ghr gene is expressed in mouse adipocytes and growth hormone has an antilipogenic effect on mouse adipose tissue (34), this is certainly a reasonable candidate gene to evaluate at the Do3 locus on chromosome 15 .

The AKR/J $\times S W R / J$ model of differential sensitivity to dietary obesity is a unique model to study the interaction of genes with dietary environment. The AKR/J mice significantly increase body fat content on a diet high in calories from fat while the SWR/J mice remain lean. Intercrosses of these two strains show that this trait segregates with complex genetics. In a previous report we have described the linkage of this trait with a genetic locus on chromosome 4 . This additional report now describes the linkage of sensitivity to dietary obesity to two additional loci on chromosomes 9 and 15. Several interesting candidate genes have been mapped to these three genetic loci and additional studies of the genetics and physiology of these candidate genes will be required to determine if they are involved in the control of this phenotype.

\section{Acknowledgments}

We thank Jody Waguespack and Stephanie Hancock for technical assistance and Dr. Pat Wozniak for consulting on the statistical design.

\section{References}

1. Kissebah, A. H., D. S. Freedman, and A. N. Peiris. 1989. Health risks of obesity. Med. Clin North Am. 73:111-138.

2. Stunkard, A. J., T. T. Foch, and Z. Hrubec. 1986. A twin study of human obesity. JAMA (J. Am. Med. Assoc.) 256:51-54.

3. Moll, P. P., T. L. Burns, and R. M. Lauer. 1991. The genetic and environmental sources of body mass index variability: the muscatine ponderosity family study. Am. J. Hum. Genet. 49:1243-1255.

4. Price, R. A. 1987. Genetics of human obesity. Ann. Behav. Med. 9:9-14.

5. Dreon, D. M., B. Frey-Hewitt, N. Ellsworth, P. T. Williams, R. B. Terry, and P. D. Wood. 1988. Dietary fat:carbohydrate ratio and obesity in middle-aged men. Am. J. Clin. Nutr. 47:995-1000.

6. Curb, D. J., and E. B. Marcus. 1991. Body fat and obesity in Japanese Americans. Am. J. Clin. Nutr. 53:1552S-1555S.

7. Copeland, N. G., N. A. Jenkins, D. J. Gilbert, J. T. Eppig, L. J. Maltais, J. C. Miller, W. F. Dietrich, A. Weaver, S. E. Lincoln, R. G. Steen, L. D. Stein, J. H. Nadeau, and E. S. Lander. 1993. A genetic linkage map of the mouse: current applications and future prospects. Science (Wash. DC). 262:57-66.

8. Bray, G. A., and D. A. York. 1979. Hypothalamic and genetic obesity in experimental animals: an autonomic and endocrine hypothesis. Physiol. Rev. 59:719-809.

9. West, D. B., C. N. Boozer, D. L. Moody, and R. L. Atkinson. 1992. Obesity induced by a high fat diet in nine strains of inbred mice. Am. J. Physiol. 262:R1025-R1032.

10. West, D. B., J. Waguespack, B. York, J. Goudey-Lefevre, and R. A. Price. 1994. Genetics of dietary obesity in AKR/J $\times$ SWR/J mice: segregation of the trait and identification of a linked locus on chromosome 4. Mamm. Genome. In press.

11. Lander, E. S., and D. Botstein. 1986. Mapping complex genetic traits in humans: new methods using a complete RFLP linkage map. Cold Spring Harbor Symp. Quant. Biol. 51:49-62.

12. Lander, E. S., and D. Botstein. 1986. Strategies for studying heterogeneous genetic traits in humans by using a linkage map of restriction fragment length polymorphism. Proc. Natl. Acad. Sci. USA. 83:7353-7357.

13. Dietrich, W., H. Katz, S. E. Lincoln, H. S. Shin, J. Friedman, N. Dracopoli, and E. S. Lander. 1992. A genetic map of the mouse suitable for typing intraspecific crosses. Genetics. 131:423-447.

14. Paterson, A. H., J. W. DeVerna, B. Lanini, and S. D. Tanksley. 1990. Fine mapping of quantitative trait loci using selected overlapping recombinant chromosomes in an interspecies cross of tomato. Genetics. 124:735-742.

15. Jacob, H. J., K. Lindpaintner, S. E. Lincoln, K. Kusumi, R. K. Bunker, Y-P. Mao, D. Ganten, V. J. Dzau, and E. S. Lander. 1991. Genetic mapping of a gene causing hypertension in the stroke-prone spontaneously hypertensive rat. Cell. 67:213-224.

16. Warden, C. H., J. S. Fisler, M. J. Pace, K. L. Svenson, and A. J. Lusis. 1993. Coincidence of genetic loci for plasma cholesterol levels and obesity in a multifactorial mouse model. J. Clin. Invest. 92:773-779.

16a. West, D. B., B. A. York, J. Goudey-Lefevre, and G. E. Truett. 1994. Genetics and physiology of dietary obesity in the mouse. In Pennington Center Nutrition Series, Vol. 5. Molecular and Genetic Aspects of Obesity. G. A. Bray and D. Ryan, editors. Louisiana State University Press, Baton Rouge. In press.

17. Labarc, C., and K. Paigen. 1980. A simple, rapid and sensitive DNA assay procedure. Anal. Biochem. 102:344-352.

18. Lander, E. S., and D. Botstein. 1989. Mapping mendelian factors underlying quantitative traits using RFLP linkage maps. Genetics. 121:185-199.

19. Lander, E. S., P. Green, J. Abrahamson, A. Barlow, M. J. Daly, S. E. Lincoln, and L. Newburg. 1987. MAPMAKER: An interactive computer package 
for constructing primary genetic linkage maps of experimental and natural populations. Genomics. 1:174-181.

20. DiVicente, M. C., and S. D. Tanksley. 1993. QTL analysis of transgressive segregation in an interspecific tomato cross. Genetics. 134:585-596.

21. Bjorntorp, P. 1992. Abdominal fat distribution and disease: an overview of epidemiological data. Ann. Med. 24:15-18.

22. Fisler, J. S., C. H. Warden, M. J. Pace, and A. J. Lusis. 1993. BSB: new mouse model of multigenic obesity. Obesity Res. 1:271-280.

23. Blatt, C., P. Eversole-Cire, V. H. Cohn, S. Zollman, R. E. K. Fournier,

L. T. Mohandas, M. Nesbitt, T. Lugo, D. T. Jones, R. R. Reed, L. P. Weiner, R. S. Sparkes, and M. I. Simon. 1988. Chromosomal localization of genes encoding guanine nucleotide-binding protein subunits in mouse and human. Proc. Natl. Acad. Sci. USA. 85:7642-7646.

24. Birnbaumer, L., J. Abramowitz, and A. M. Brown. 1990. Receptor-effector coupling by G proteins. Biochim. Biophys. Acta. 1031:162-224.

25. Lin, C. K., M. J. Hakakha, J. M. Nakamoto, A. T. Englund, A. S. Brickman, M. L. Scott, and C. Van Dop. 1992. Prevalence of three mutations in the $\mathrm{G}_{\mathrm{s} \text {-alph }}$ gene among 24 families with pseudohypoparathyroidism type Ia. Biochem. Biophys. Res. Commun. 189:343-349.

26. Begin-Heick, N. 1992. Alpha-subunits of $\mathrm{Gs}$ and $\mathrm{Gi}$ in adipocyte plasma membranes of genetically diabetic (db/db) mice. Am. J. Physiol. 263:C121-129.

27. Murayana, T., and M. Ui. 1983. Loss of the inhibitory function of the guanine nucleotide regulatory component of adenylate cyclase due to its ADP ribosylation by islet-activating protein, pertussis toxin, in adipocyte membranes. J. Biol. Chem. 258:3319-3326.

28. Barton, D. E., B. E. Foellmer, W. I. Wood, and U. Francke. 1989. Chromosome mapping of the growth hormone receptor gene in man and mouse. Cytogenet Cell Genet. 50:137-141.

29. Eicher, E. M., and B. K. Lee. 1991. Growth hormone receptor (Ghr) and hemoglobin alpha-chain pseudogene 3(Hba-ps3) map proximal to the myelocytomatosis oncogene (Myc) on mouse chromosome 15. Mamm. Genome. 1:57-58.

30. Gregory, J. W., S. A. Greene, R. T. Jung, C. M. Scrimgeour, and M. J. Rennie. 1991. Changes in body composition and energy expenditure after six weeks' growth hormone treatment. Arch. Dis. Child. 66:598-602.

31. Flint, D. J., and M. J. Gardner. 1993. Influence of growth hormone deficiency on growth and body composition in rats: site-specific effects on adipose tissue development. J. Endocrinol. 137:203-211.

32. Searle, T. W., J. D. Murray, and P. J. Baker. 1992. Effect of increased production of growth hormone on body composition in mice: transgenic versus control. J. Endocrinol. 132:285-291.

33. Amselem, S., P. Duquesnoy, B. Duriez, F. Dastot, M. L. Sobrier, S. Valleix, and M. Goossens. 1993. Spectrum of growth hormone receptor mutations and associated haplotypes in Laron Syndrome. Hum. Mol. Genetics. 2:355-359.

34. Ng, F. M., N. A. Adamafio, and J. E. Graystone. 1990. Effects of exogenous growth hormone on lipid metabolism in the isolated epididymal fat pad of growth hormone-deficient little mouse. J. Mol. Endocrinol. 4:43-49. 